\title{
A comparative study of additive manufacturing techniques: Residual stress and microstructural analysis of CLAD and WAAM printed Ti-6AI-4V components
}

Blanka A. Szosta ${ }^{\mathrm{a},}$, Sofiane Terzi ${ }^{\mathrm{a}, \mathrm{d}}$, Filomeno Martina ${ }^{\mathrm{b}}$, Didier Boisselier ${ }^{\mathrm{c}}$, Anastasiia Prytuliak ${ }^{\mathrm{a}, \mathrm{d}}$, Thilo Pirling ${ }^{d}$, Michael Hofmann ${ }^{e}$, David J. Jarvis ${ }^{a}$

\footnotetext{
${ }^{a}$ European Space Agency, ESTEC, Keplerlaan 1, PO Box 299, NL 2200 AG Noordwijk, The Netherlands

${ }^{\mathrm{b}}$ Cranfield University, Cranfield, Bedfordshire MK43 OAL, UK

' Irepa Laser, Parc d'Innovation - Pôle API, Illkirch 67400, France

dInstitut Laue-Langevin, CS 20156, F-38042 Grenoble Cedex 9, France

${ }^{e}$ FRMII TU Munich, Lichtenbergstrasse 1, D-85747 Garching, Germany
}

\section{Abstract}

Nowadays, there is a great manufacturing trend in producing higher quality net-shape components of challenging geometries. One of the major challenges faced by additive manufacturing (AM) is the residual stresses generated during AM part fabrication often leading to unacceptable distortions and degradation of mechanical properties. Therefore, gaining insight into residual strain/stress distribution is essential for ensuring acceptable quality and performance of high-tech AM parts. This research is aimed at comparing microstructure and residual stress built-up in Ti-6Al-4V AM components produced by Wire + Arc Additive Manufacturing (WAAM) and by laser cladding process (CLAD).

\section{Introduction}

Additive manufacturing, often called 3D printing is nowadays among the most studied processes. AM is a key technique of a great potential in reducing high cost of producing conventional components made from relatively expensive materials such as titanium alloys. The worldwide Ti component production is constrained due to the high cost of $\mathrm{Ti}$ in comparison to other materials. Therefore, AM techniques aiming towards zero waste manufacturing are identified as potential prosperous routes in broadening Ti parts fabrication that are usually affected by often difficult and extensive machining. Ti is very broadly used in space, aerospace, nuclear, marine and chemical industries by virtue of its desirable properties such as high specific strength combined with excellent corrosion and oxidation resistance [1]. Although $\mathrm{Ti}$ is a very cherished material, its use in $\mathrm{AM}$ processes is also relatively challenging because of its low thermal conductivity which results in drawbacks such as uneven temperature field and poor interlamination integration [2]. Avoiding extensive machining by a near netshape successive layers fabrication can reduce the Ti parts production cost significantly. The buy-tofly ratio for a part machined from forged billet is typically 10-20 [3] and can potentially drop to nearly 1 when produced by AM techniques. There are numerous AM techniques that are capable of producing complex geometries close to their net-shape. Simply, AM techniques can be classified according to feeding technique, heat source or feedstock material. Powder bed, blown power and wire feed are the main AM techniques using heat sources such as electron beam, laser or electric arc, while the most common feedstock materials are powder or wire. Despite the similarity of AM 
techniques, they are often named differently as they are branded by various manufactures, notwithstanding all of them fall under one from the mentioned categories. Wire-fed techniques are often called Shaped Metal Deposition (SMD) [4] or Wire + Arc Additive Manufacturing (WAAM) [5,6] when an electric arc is used as a wire melting source. As for the powder-based technology, usually a laser or an electron beam is used as melting power source. Among the most studied, we can mention: Selected Laser Melting (SLM) and Electron Beam Melting (EBM) $[7,2]$ for the powder-bed processes and Laser Metal Deposition (LMD) or laser cladding (CLAD) [8] for the blown-powder processes. EBM process is often used for an industrial fabrication of Ti-6Al-4V parts due to its moderate operation costs, high energy efficiency; and higher scan speed ( 500 mm s-1) comparing to the WAAM and CLAD methods. However, EBM AM part size is determined by the powder bed geometry, and therefore for the larger parts production WAAM or CLAD techniques are preferred [9]. AM process parameters are specific to the alloy feedstock and AM technique. Therefore, in the first place the manufacturers usually conduct parametric studies in order to determine the best sets of process parameters and optimise the geometry, cost and overall properties of the AM parts. The parameters such as process stability, alloy feedstock quality, energy efficiency, energy density, scan speed, process environment, clamping strategy and scanning build path are the most frequently studied and discussed by the additive manufacturing community $[6,8,10,11,12]$. Unfortunately, per contra to great prospects in net-shape manufacturing all AM techniques must deal with several issues; like lack of fusion, cracking, vaporisation, high porosity level, uncontrolled grain size, distortions and residual stress, with the latter being one of the most crucial in determining the component performance. Additive manufacturers $[13,5,14,15]$ have been trying to eliminate these problems by extensive study of AM process parameters targeting simultaneously the economical and environmental benefits of additive over conventional manufacturing. These benefits are closely linked to deposition rates capability that also determines geometrical accuracy and surface finish, therefore achieving a specific target requirement is challenging [14]. The AM process parameters investigated in the literature are for instance: laser pulse length and duty cycle that showed slight influence on residual stresses in Waspaloy printed by laser direct metal deposition [13]. Another example that is worth to mention is high pressure rolling introduced in WAAM process that was proven to reduce residual stresses in steel components [5]. Ti-6AI-4V AM components were previously studied, however these investigations were mostly focused on changes that occur according to the AM process parameter variations mostly in one system [16]. However, certain AM techniques may be more suitable for fabricating specific components rather than the other. Comparison of the same geometry parts produced by different methods is crucial for modelling, creating benchmarking and standards that still need to be established for these relatively new manufacturing routes. Detailed studies and comparison of AM processes and their parameters are important for understanding the physics of AM and determining all variables that influence the final results. Not many studies on residual stresses in titanium components produced by different techniques were examined and compared one another. The present study was undertaken to investigate these differences in terms of residual stresses generated and microstructure, in the case of $\mathrm{Ti}$ (T-shape) samples produced by two different AM techniques. 


\section{Material and methods}

\subsection{Material, AM techniques and procedure}

A commercial Ti alloy, Ti-6Al-4V, was chosen for this study. The chemical composition of Ti-6Al-4V is shown in Table 1.

Table 1 - Chemical composition of the feed material used for the additive processing (wt.\%).

\begin{tabular}{|l|r|r|r|r|r|r|r|r|r|}
\hline & $\mathbf{T i}$ & $\mathbf{A l}$ & $\mathbf{V}$ & $\mathbf{F e}$ & $\mathbf{O}$ & $\mathbf{C}$ & $\mathbf{N}$ & $\mathbf{H}$ & Others \\
\hline Powder & Bal. & 6.14 & 3.95 & 0.19 & 0.13 & 0.02 & 0.010 & 0.001 & $<0.16$ \\
\hline Wire & Bal. & 6.14 & 3.96 & 0.18 & 0.14 & 0.02 & 0.011 & 0.001 & $<0.15$ \\
\hline
\end{tabular}

Ti-6Al-4V was used in form of wire or powder in the WAAM and CLAD samples, respectively. Both specimens were produced in similar dimensions and were deposited on similar baseplates (Ti-6Al$4 \mathrm{~V}$ rolled plates) clamped in the same way. In wire-fed technique a Lincoln Electric Invertec V310-T TIG power supply was used to deposit titanium wire onto the substrate (sample provided by Cranfield University). 40 layers of approximately $250 \mathrm{~mm}$ long, $5.9 \mathrm{~mm}$ wide and $1.0 \mathrm{~mm}$ high were deposited on a $250 \times 60 \times 7 \mathrm{~mm}$ baseplate with a travel speed of $4.5 \mathrm{~mm} / \mathrm{s}$; the wire feed speed was $1.6 \mathrm{~m} / \mathrm{min}$; the average current was $110 \mathrm{~A}$ with a pulse duration of $0.05 \mathrm{~s}$ and a frequency of $10 \mathrm{~Hz}$; a local trailing shield which blew argon gas was used to prevent oxidation of the deposited material. In laser blown-powder process, Easy CLAD MAGIC LF6000 laser was used in argon $\left(\mathrm{O}_{2}<15 \mathrm{ppm}\right.$ and $\mathrm{H}_{2} \mathrm{O}<40 \mathrm{ppm}$ ) atmosphere with shielding argon gas at the travel speed of $33.3 \mathrm{~mm} / \mathrm{s}$ and feed speed of $0.245 \mathrm{~g} / \mathrm{s}$ (sample provided by Irepa Laser). To reach comparable wall thickness (of about $7.5 \mathrm{~mm}$ ), four tracks were used according to the deposition procedure described in Fig. 1. 65 layers of approximately $0.85 \mathrm{~mm}$ high and $250 \mathrm{~mm}$ long were thus deposited on a $250 \times 60 \times 8 \mathrm{~mm}$ baseplate following this method.

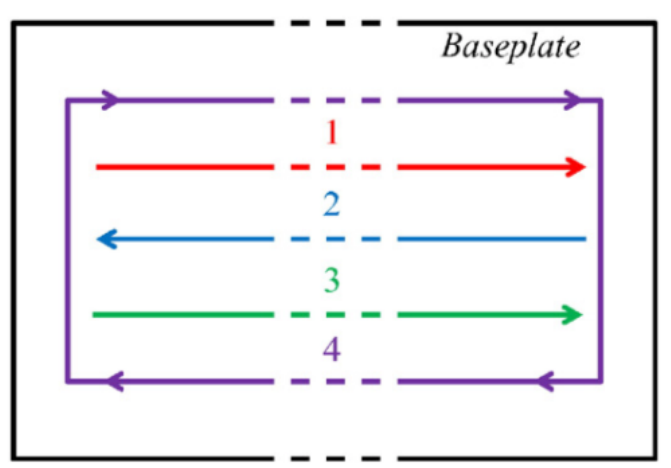

Figure 1 - Laser deposition path to build the CLAD sample: three parallel beads were deposited first parallel to each other following by a fourth shell-bead deposited around them.

\subsection{Residual microstrain and stress measurement}

Neutron diffraction is a non-destructive technique particularly adapted for determining 3D residual stresses within bulk materials and engineering components owing to the high penetrating power of 
neutron beams [17]. The principle behind neutron residual stress analysis is the determination of the variation in diffraction angle, which is attributed to a change in lattice parameter owing to tensile or compressive stress within the material. The average strain in the gauge volume sampled for a given direction and particular \{hkl\} crystallographic planes can be calculated using [18]:

$$
\varepsilon_{h k l}=\frac{d_{h k l}-d_{0, h k l}}{d_{0, h k l}}=\frac{\sin \theta_{0, h k l}}{\sin \theta_{h k l}}(1)
$$

where $d_{h k l}$ is the lattice plane spacing and $\theta_{\text {hkl }}$ the angle in between the incident beam and the scattering planes $\{h \mathrm{hl}\}$. $\mathrm{d}_{0, \mathrm{hkl}}$ and $\theta_{0, \mathrm{hkl}}$ are the reference of values for the stress-free material. In many cases it is often difficult to directly measure strain free lattice spacing and reference values are thus calculated. There are various methods available to estimate $d_{0, \mathrm{hkl}}$ and $\theta_{0, \mathrm{hkl}}$. In the present study, we used the mean value of $\theta_{\text {nkl }}$ averaged over the height of the sample in the three principal directions [19]. A comparison with the other methods can be found in the Appendix A. The principal stresses can then be calculated by using the triaxial form of Hooke's Law:

$$
\theta_{i i}=E_{h k l} \frac{\left(1-v_{h k l}\right) \varepsilon_{i i, h k l}+v_{h k l}\left(\varepsilon_{j j, h k l}+\varepsilon_{k k, h k l}\right)}{\left(1+v_{h k l}\right)\left(1-2 v_{h k l}\right)}
$$

where $E_{\mathrm{hkl}}$ is the Young modulus of titanium assumed to be $105.5 \mathrm{GPa}$ for the $\{\mathrm{hkl}\}$ planes used [20], Vhkl the Poisson ratio equal to 0.34 and $\varepsilon_{x x, h k l}, \varepsilon_{y y}, h k l, \varepsilon_{z z z, h k l}$ the strains measured in the three principle directions. The uncertainties $\pm \Delta \varepsilon_{\mathrm{ii}, \mathrm{hkl}}$ and $\pm \Delta \sigma_{\mathrm{ii}}$ are given by:

$$
\begin{gathered}
\Delta \varepsilon_{i i, h k l}=\sqrt{\frac{d_{0, h k l}^{2} \Delta d_{i i, h k l}^{2}+d_{i, h k l}^{2} \Delta d_{0, h k l}^{2}}{d_{0, h k l}^{4}}}(3) \\
\Delta \sigma_{i i}=\frac{E_{h k l} \sqrt{\left(v_{h k l}-1\right)^{2} \Delta \varepsilon_{i i, h k l}^{2}+v_{h k l}^{2}\left(\Delta \varepsilon_{j j, h k l}^{2}+\Delta \varepsilon_{k k, h k l}^{2}\right)}}{\left(1+v_{h k l}\right)\left(1-2 v_{h k l}\right)}
\end{gathered}
$$

Residual stress measurements by neutron diffraction were performed at FRM II Heinz Maier-Leibnitz in Garching, Germany. The instruments parameters used are listed in Table 2. For each point, the scan time was minimum $15 \mathrm{~min}$. In order to perform the measurement in the three principal directions and to elude the beam attenuation passing by the whole baseplate width, several alignments were required. 
Table 2 - Neutron diffraction measurement parameters; gauge volume measured in (a) transverse and normal direction and (b) in longitudinal direction.

\begin{tabular}{|r|r|r|r|r|r|}
\hline Instrument & Wavelenght (A) & $\begin{array}{r}\text { Detector angle } \\
\text { (degrees) }\end{array}$ & Plane & $\begin{array}{r}\text { Monochromator } \\
\text { Gauge Volume } \\
\left(\mathbf{m m}^{3}\right)\end{array}$ \\
\hline StressSpec & -1.83 & -85 & $\alpha-\mathrm{Ti}(103)$ & $\mathrm{Si}(400)$ & $3 \times 2 \times 20^{(\mathrm{a})}$ \\
& & & & & $3 \times 3 \times 2^{(\mathrm{b})}$ \\
\hline
\end{tabular}

\subsection{Optical microscopy and distortion measurements}

The samples were cut in transverse and longitudinal directions, cold mounted in curing epoxy (CaldoFix-2), ground using 1200 grit SiC paper then polished with $6 \mu \mathrm{m}, 3 \mu \mathrm{m}$ diamond suspensions, and finally polished with a mixture of colloidal silica (OP-S) containing $30 \%$ hydrogen peroxide. The samples were etched in Kroll's reagent (5\% hydrofluoric acid, $10 \%$ nitric acid and $85 \%$ distilled water) and observed by using a Leica DMI 5000M optical microscope. The out-of-plane baseplate distortion measurement (D) was carried out by using a Mitutoyo 500-196-20 digital calliper as shown in Fig. 2 .

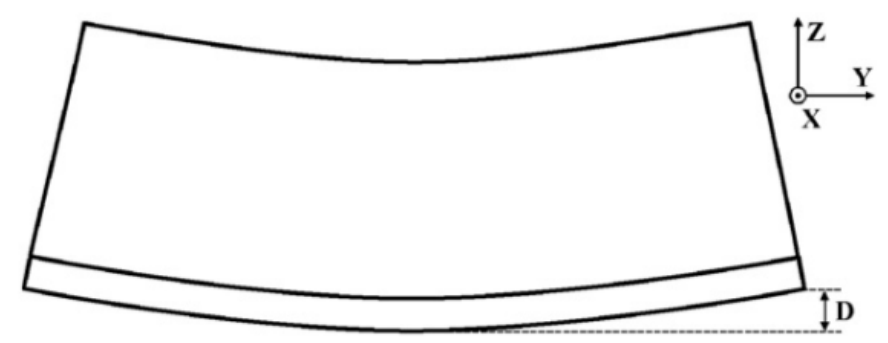

Figure 2 - Out-of-plane baseplate distortion measurements (D)

\section{Results}

\subsection{Distortion, sample shape and defect}

In the WAAM sample $\sim 7.2 \mathrm{~mm}$ out-of-plane distortion was measured while the sample produced by CLAD process showed $\sim 3.5 \mathrm{~mm}$ of distortion. Optical microscopy done on tested AM specimens exposed highly dense structures almost porosity and underfill free (Fig. 3). Only few pores were detected near the bottom region of the wall. (Fig. 4). The surface profile and roughness of the wall in cross section can be qualified in terms of deviation from the surface of the ideal form (here a vertical flat wall). Deviation at short wavelength can be observed in both samples. They correspond to the different layers deposited on top of each other. The periods are about $0.85 \mathrm{~mm}$ for the CLAD sample and $1 \mathrm{~mm}$ for the WAAM sample. Their amplitudes are similar in both samples but the shape of the ripples is smoother in the sample manufactured by WAAM. However, in the case of the CLAD sample, the verticality and parallelism of the wall surfaces are remarkable while long wavelength ripples can be observed in the case of the WAAM sample but their amplitude remains low. It can also be noticed that the bottom of the walls produced by WAAM technique shows slight necking in the vicinity of the baseplate (due to thermal effect as discussed in [21]) and that the top of the sample is rounded by contrast with the CLAD sample which shows a flatter top. 


\subsection{Microstructure}

Etching and optical microscopy conducted on the cross sections of the studied samples revealed heat-affected zones (HAZs) in the transition region in between the deposited wall and the substrate (Fig. 5). The CLAD sample showed a HAZ of approximately $8.5 \mathrm{~mm}$ in width and $\sim 0.7 \mathrm{~mm}$ in depth, while the WAAM specimen showed HAZ of $\sim 9.3 \mathrm{~mm}$ wide and $\sim 2.7 \mathrm{~mm}$ deep. The microstructure of both samples exhibited typical Widmanstätten two-phase morphology composed of (BBC) prior $\beta$ grains1 and (HCP) acicular $\alpha$ phases in form of colony or basketweave. $\alpha$ phases were also visible at $\beta$-grain boundary in particular in the WAAM specimen.

In both samples, small equiaxed $\beta$-grains (30-100 $\mu \mathrm{m})$ are visible in the HAZ. The size and shape of $\beta$-grains then evolves differently in the two samples. The microstructure of the CLAD sample showed narrow columnar grains of 100 to $300 \mu \mathrm{m}$ width and $1 \mathrm{~mm}$ to $3 \mathrm{~mm}$ length in the bottom of the wall and then slightly larger grains up to few millimetres in the top section. A tendency to exhibit a wavy shape particularly in the middle of the wall thickness can also be observed. In the sample manufactured by WAAM process the first few layers of the wall consist of a mixture of elongated grains ranging from $\sim 500 \mu \mathrm{m}$ to $5 \mathrm{~mm}$. Then large columnar grain spreading up to the top of the sample can be observed. Layer bands can also be observed in both samples. Their structure will be addressed in detail in the discussion part. 


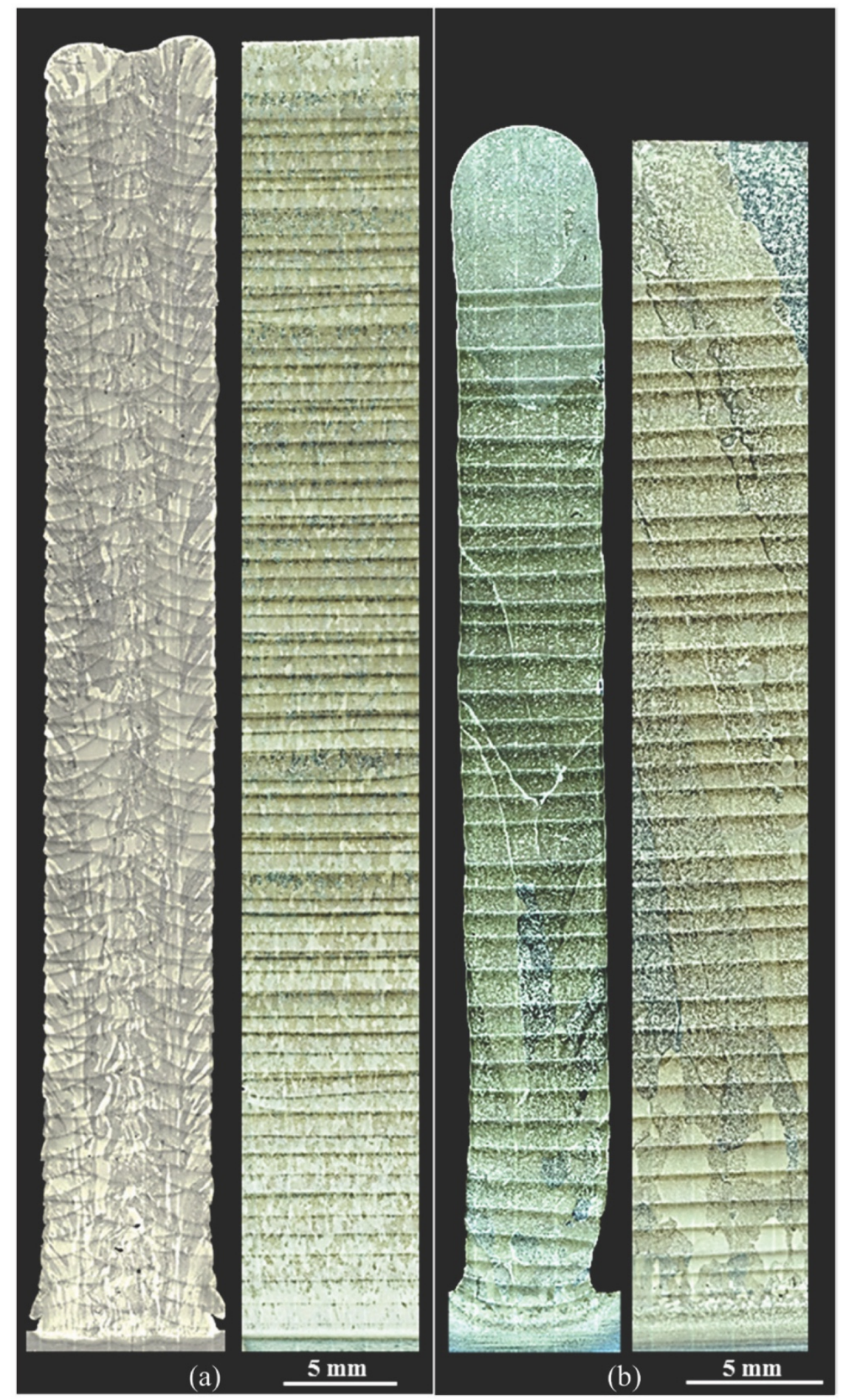

Figure 3 - Macrographs of the (a) CLAD and (b) WAAM samples. In (a) and (b), the left pictures correspond to the transversal direction and the right pictures, the longitudinal direction.

\subsection{Neutron residual microstrain and stress measurements}

The comparison of microstrain and residual stress are presented in Fig. 6. Both samples exhibit a maximum tensile stress near the baseplate-wall transition in longitudinal direction. The higher value was obtained for the WAAM sample with a peak at $480 \mathrm{MPa}$ while the maximum reached in the CLAD sample was about $280 \mathrm{MPa}$. These results vary with a strain free lattice spacing determination 
method applied. A comparison of maximum and minimum stress values depending on the chosen method is described in the Appendix A. The main difference in microstrain and residual stress are in the vicinity of the baseplate and the top of the wall, where residual stresses are more tensile and more compressive, respectively, in the sample produced by WAAM technique. The stress/microstrain in longitudinal direction on the top of the wall was significantly more compressive in as-deposited WAAM specimen. The microstrains and residual stresses in other directions are similar aside from stress in normal direction in sample produced by CLAD process, where the stresses were more compressive in the baseplate as well as the first deposited layers. The interior of CLAD sample wall accumulated tensile stress similarly to WAAM specimen.

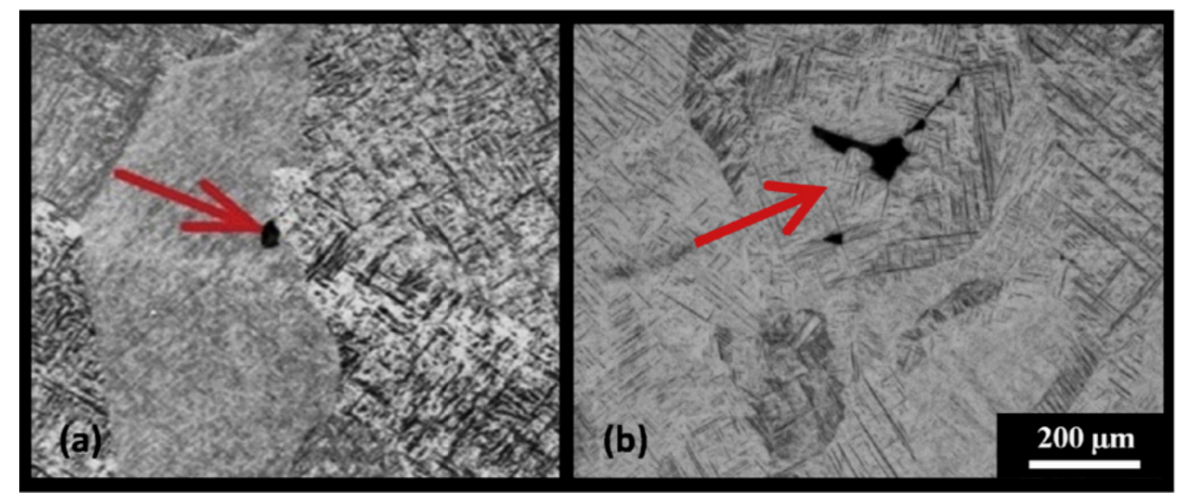

Figure 4 - Observation of pores in (a) WAAM and (b) CLAD samples.

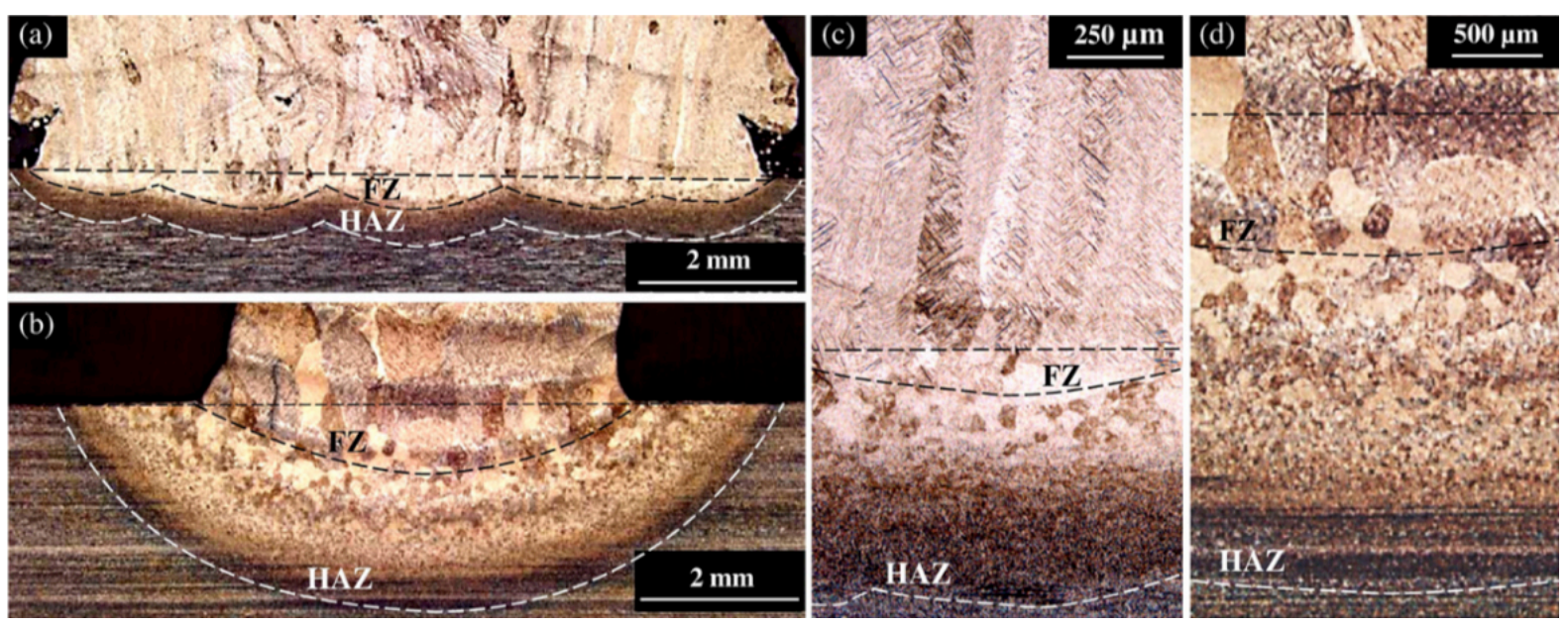

Figure 5 - Fusion zone, heat affected zone and beta grain size at the baseplate-wall interface region; (a) and (d) CLAD sample, (b) and (c) WAAM sample. 


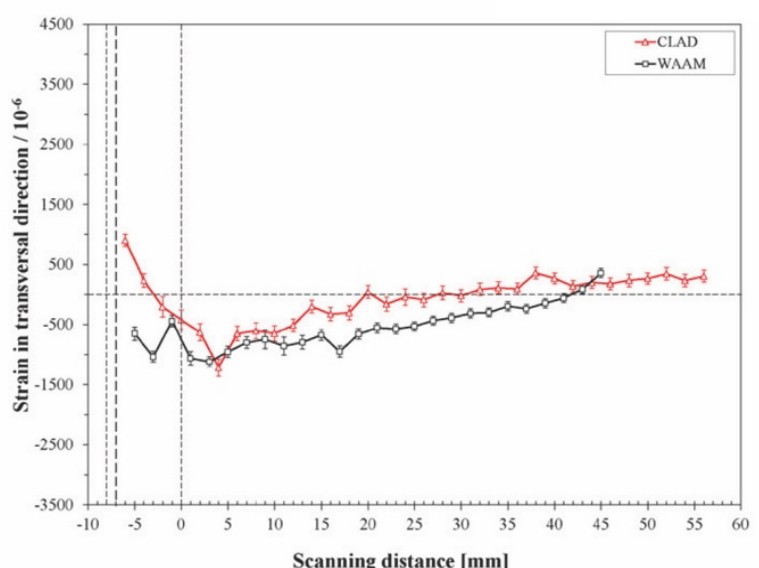

(a) Strain $\varepsilon_{x x, 103}$ in transversal direction

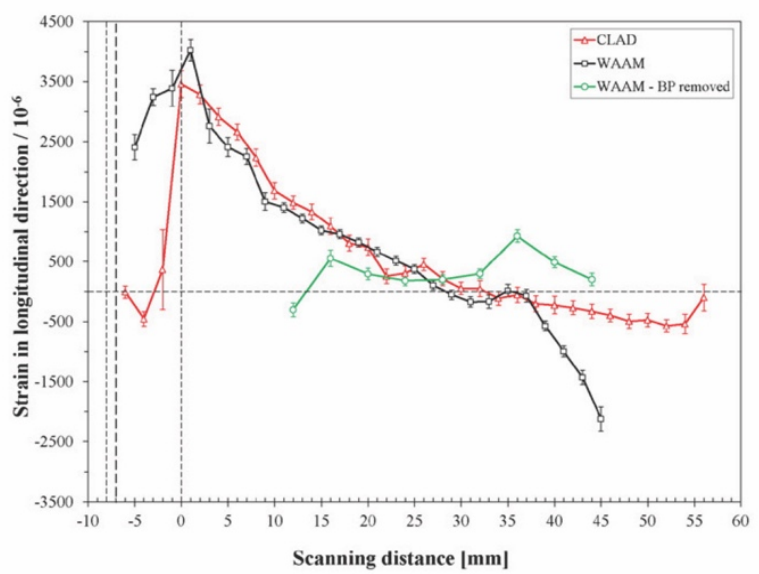

(c) Strain $\varepsilon_{y y, 103}$ in longitudinal direction

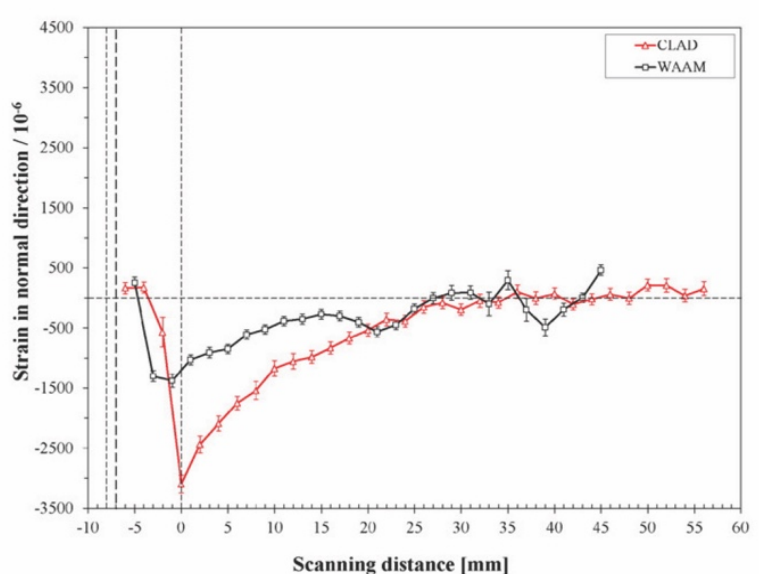

(e) Strain $\varepsilon_{z z, 103}$ in normal direction

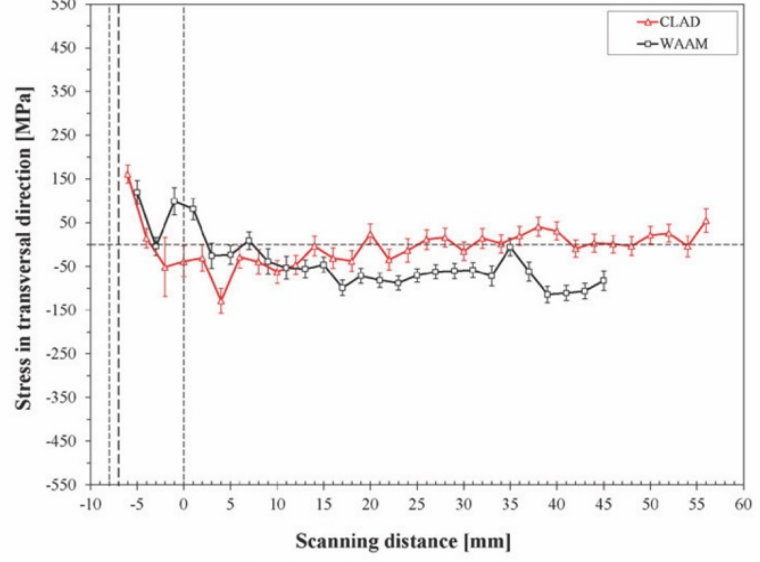

(b) Stress $\sigma_{x x}$ in transversal direction

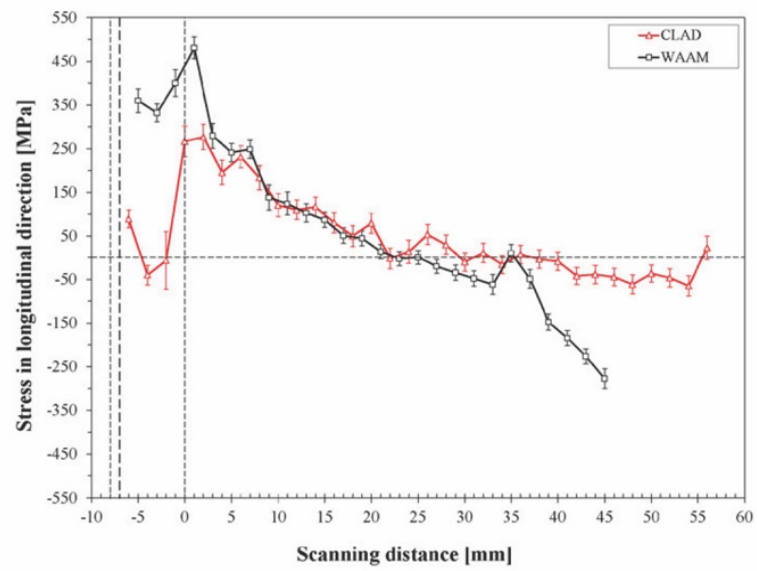

(d) Stress $\sigma_{y y}$ in longitudinal direction

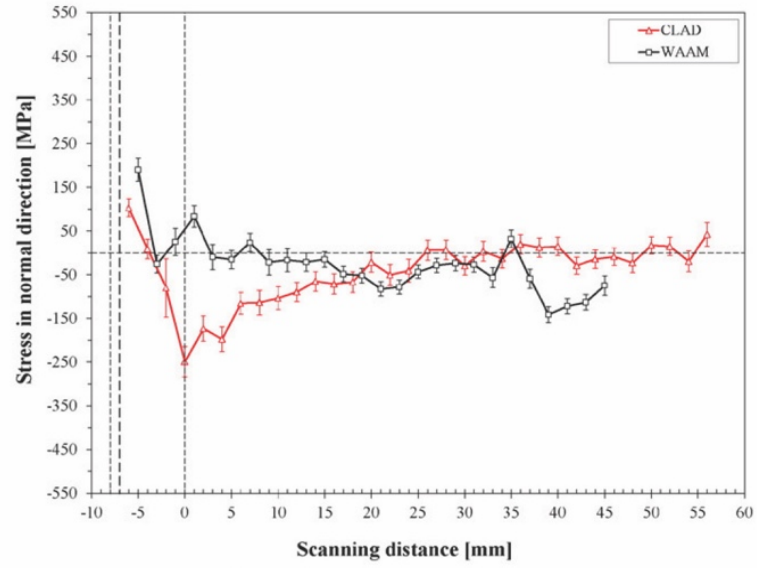

(f) Stress $\sigma_{z z}$ in normal direction

Figure 6 - Strain (left column) and stress (right column) measured by neutron diffraction. 


\section{Discussion}

\subsection{Microstructure}

In the AM processes considered here, upon deposition of the first layer, a part of the substrate is remelted forming a fusion zone (FZ) below which a HAZ is created. The extent of these layers depends on the heat input provided by the heat source as well as on the kinetic of the heat extraction that is a function of the thermal properties of materials, the environment and also on the overall geometry of the AM part (wall and baseplate). In the baseplate the heat extraction occurs radially to the melt pool in the three directions $X, Y$ and $Z$ leading to curved HAZ in transversal micrographs. In the CLAD sample, multiple HAZ could be seen as several parallel beads were deposited to reach a wall thickness similar to the WAAM sample. The HAZ can potentially extends to regions that have seen a peak temperature as low as the martensite start temperature $M_{s}$ where the $\beta$ phase can transform into martensite under high cooling rate. No significant change in microstructure is expected below the dissolution temperature of the $\alpha$-phase $\left(T_{\text {Diss }}=981 \mathrm{~K}\right)$ unless the cooling rate exceeds the critical value for martensite transformation. In the regions that have seen a temperature in between the dissolution temperature and the $\beta$-transus, changes in the morphology of the $\alpha$-phase are expected. In etched microstructures, the HAZ limit can be noticed from the difference in contrast with non-affected zones in the baseplate. Clearly the HAZ was much deeper and wider in the WAAM sample. As the substrates had similar geometries and thermal properties, the differences can mostly be attributed to the heat input and the size of the melt pool that were more significant in the WAAM specimen. In the parts of the substrate that saw temperatures higher than the $\beta$-transus ring the deposition process, the initial characteristics of the $\alpha$-phase are totally erased. Such event can then occur several times upon subsequent layer deposition. Between the $\beta$-transus and the solidus, the growth of $\beta$-grains becomes activated and coarsening of the baseplate grains can occur in HAZ. This takes place at each excursion in the one phase region above the $\beta$-transus. The resulting outcome is a gradual increase in grain size towards regions where the time spent at high temperature was longer. Both samples exhibited this coarsening-zone but for the same reason as mentioned before, in the CLAD sample it was narrower and the maximum grain size reached appeared smaller. During 3Dprinting, when the first layer is deposed, a part of the baseplate also remelts and joins the melt pool with the added material. If the baseplate and feeding material are identical and a strong thermal gradient is present, the beginning of solidification is directional and occurs epitaxially from the substrate providing that the feeding material is totally remelted. The baseplate acts as an ideal substrate and solidification often kicks off without nucleation barrier and undercooling [22]. The partially-remelted grains of the substrate start to regrowth in opposite direction of the heat flow. A grain selection process then occurs where grains that are oriented in the most favourable direction grow faster while the others tend to be overgrown. Both CLAD and WAAM samples showed an onset of directional growth, characterised by the columnar grains occurring in the baseplate region.

Depending on process conditions, the time needed to establish the columnar growth can vary. This can leave a layer of variable size, below clearly elongated grains, where overgrown grains of lower aspect ratio can be seen. This region marks the beginning of the FZ which can sometimes be difficult to distinguish from the area in the HAZ where significant grain coarsening takes place. Note that 
under certain conditions (e.g. with a high level of nucleants), the columnar to equiaxed transition (CET) can occur early in the solidification process and the columnar/dendritic growth is restricted. Newly nucleated equiaxed grains can thus be seen in the FZ of the baseplate but in that case a subsequent regression towards columnar growth does not generally occur. Indeed, with the local decrease of liquid fraction the ratio $G / V$ (where $G$ is the thermal Gradient and $V$ the growth velocity) tends to decrease which drives the solidification path towards the equiaxed growth domain [23].

Both CLAD and WAAM specimen exhibited columnar grains in the middle region of the sample with a tendency to grow outward approaching the sample surface in agreement with the evolution of the liquidus curvature (see transversal section Fig. 3). However, in the CLAD samples some grains appeared wavy close to the centre line. This can be explained by the fact that multiple overlapped beads were used to build the sample. Some grains in the middle of the thickness were alternatively partially-remelted and regrown as part of the solidification process of different beads. The heat flow was then different resulting in a variation of the growth direction. This had a disturbing effect on grain selection which was likely to contribute to the difference in grain size observed between the CLAD and WAAM samples. Similar features were also reported in the literature when the travelling direction of the melt pool is alternated. It was shown that the angle of the solidification front at the melt pool tail influence the grain orientation [24]. By alternating the direction of the deposition process from one layer to another, wavy grains were observed in longitudinal section [15]. Interestingly this feature was not clearly visible in the longitudinal section of the CLAD sample while the deposition direction was also alternated. This could be due to the fact that grains exhibiting a wavy shape on one plane (i.e. transversal here) can appear as multiple smaller grains in a perpendicular plane (i.e. the longitudinal plane here). The aspect ratio being less pronounced on that plane, the change in orientation is less visible. The WAAM sample was manufactured without changing the deposition direction between layers. Tilting of the columnar grain was clearly visible and can be estimated to about 15 to $20^{\circ}$. As just mentioned, the tilting angle is a function of the melt pool shape. It was found that shallower and more elongated melt pools lead to smaller tilt angles [24]. The WAAM process lead to deeper melt pool in comparison to the CLAD process which could also explain why tilting was less visible in the latter.

One of the most noticeable features on micrographs was the presence of layer bands visible in both samples (Fig. 3). On transversal section, in the CLAD sample they appeared as multiple curved lines (several of them throughout the wall thickness due to the deposition of multiple tracks) while in the WAAM sample they appeared as continuous curved lines in the baseplate region and as straight horizontal lines in the middle section. These layer bands do not mark the limit between deposited layers. They were not coinciding with the ripples at the sample surface and they were not visible in the top regions of samples. It was found in literature that these layer bands form when the material is reheated near the $\beta$-transus without subsequent excursion at higher temperatures. Preferential conditions are present for the $\alpha$-phase to grow in form of colonies leaving locally a higher volume fraction of this type of phase morphology [25]. By looking at the top region, we can estimate the extent 
of the zone that saw a temperature higher than the $\beta$-transus upon the deposition of a new layer (Fig. 7). In the case of the CLAD sample this zone extended down to 1-2 layers below the newly deposited matter and down to 2-3 layers for the WAAM sample. Below the layer band a gradient of contrast (dark to bright here) was visible. It coincides with observations reported in the literature of a gradual decrease in size of the a-phase from top to bottom of the layer [25]. While a detailed quantitative measurement was out of the scope of the present study, qualitative observation seems to confirm this general trend. However, it should be noticed that the contrast gradient was only clearly visible close to the layer band for the CLAD sample by comparison with the WAAM where it extended further down. Moreover, layer bands (appearing in light contrast) were much thicker in the case of the WAAM sample and can be easily noticed. This is in agreement with local HAZ formed by each deposited layer, being more extended for the WAAM than for the CLAD process. However, the fact that multiple tracks were used increased the overall area affected by the heat (i.e. five curved HAZ are created for building one layer). It is also worth reminding that the observed contrast depends on various parameters such as the property of the material, etching time and observation conditions which were tried to be kept constant in this analysis.
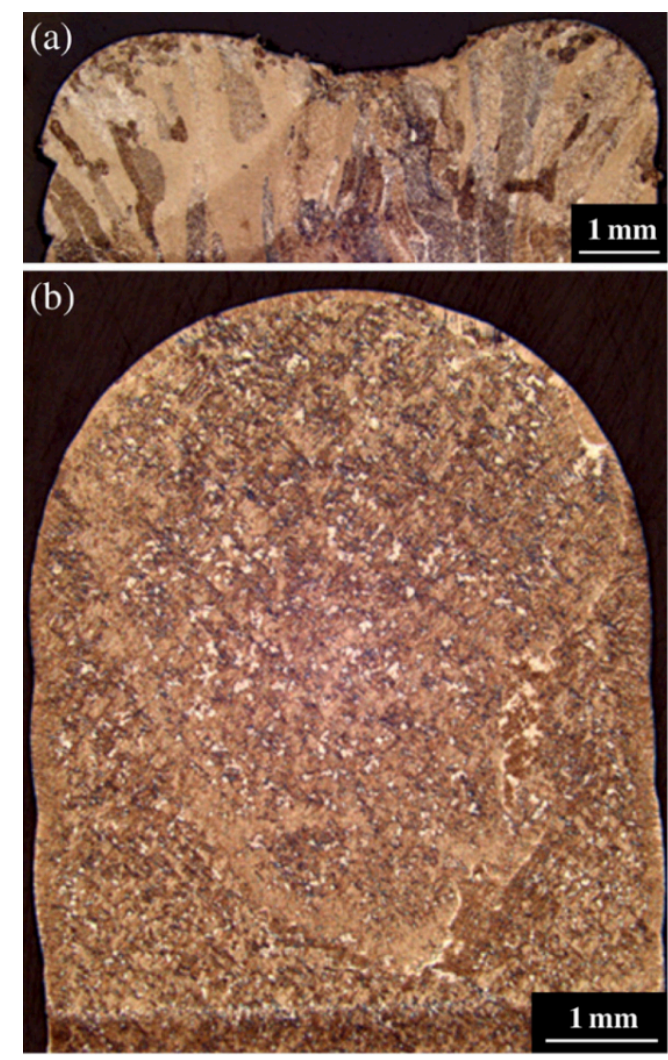

Figure 7 - Micrographs of the top layers of the (a) CLAD and (b) WAAM samples.

It should be noted that the microstructure of the Ti-6AI-4V samples produced by WAAM and CLAD is different from those observed in as-cast and wrought. Wrought alloys generally exhibit equiaxed $\alpha-\beta$ elongated grains in the rolling direction [26] while the as-cast ones usually show very coarse prior $\beta$ grains with non-uniform size distribution [27]. 


\subsubsection{Defects formation}

Porosity is one of the main problems along with crack formation upon additive process manufacturing. It can be generated by different mechanisms such as gas entrapment, gas condensation and shrinkage porosity. The gas entrapped phenomenon is associated with gas blown into the melt pool that does not have sufficient time to escape before solidification. Powder blown processes such as CLAD are therefore more susceptible to porosity formation by this mechanism than WAAM process. Multiple parallel track deposition necessary for achieving a required thickness (as it is the case for CLAD here) can also trigger additional gas entrapment located in between parallel beads by a lack of fusion of adjacent material. Few pores with typical star-shape were observed in the CLAD sample indicating pores formation resulting from multi-track powder deposition (Figs. 3a and 4). To date, there is few data available on pore formation in multibead WAAM process deposition which is surely an interesting subject of study. It can be assumed that this additional source of porosity can increase the occurrence of defects if hashing pattern is not optimised. The shape and cleanliness of substrate, the deposition strategy as well as laser/arc melt scanning velocity are critical in avoiding porosity formation by gas entrapping mechanism. In the early stage of solidification gas condensation of dissolved gas in the melt (generally hydrogen coming from moisture or contamination of the feedstock material [28]) can also form pores upon freezing as a result of the difference of solubility of such gas in the liquid and solid phase [29]. This mechanism usually leads to spherical pores as they form at low solid fraction. Shrinkage porosity on the other hand occurs at the late stage of solidification. It is due to the volume change upon solidification combined with restricted feeding of liquid to the final solidification region [29]. It appears above the dendrite coherency solid fraction ( $\left.f_{s, c o h}\right)$ when dendrite impinged and form a percolating network of solid. The porosity then grows between dendrites resulting in pores with tortuous shape. When $\mathrm{f}_{\mathrm{s}, \mathrm{coh}}$ is high the occurrence of pores by this mechanism is expected to be limited. Considering the alloy composition and solidification conditions (directional solidification at high cooling rate) this mechanism is therefore not expected to be the predominant mechanism. Pores are not the only defects in AM, other types of defects such as cracks can develop in the solidified microstructure when residual stresses are too high after material deposition.

\subsection{Residual stress, distortion and overall sample shape}

\subsubsection{Unclamping and stress redistribution}

When molten metal is deposited on a cold substrate, the thermal contraction of the solidified material occurring during solidification creates tensile stress in the deposit and compression stress in the substrate. As 3D printed structures consist of numerous welds layered on each other, locked-in residual stresses can reach a remarkable level. During the building process, however, the substrate undergoes multiple heating and cooling steps alternating phases of stress relaxation and accumulation. The magnitude and distribution of the stress reached in the build is therefore influenced by various factors such as the deposition strategy and the melt-pool size. Nevertheless, with the considered samples geometry, a general trend where stresses are more tensile in the wall and compressive in the baseplate can be predicted for the stress state reached after deposition. It can also be foreseen that the stress will be maximal in the longitudinal direction and minimal in the 
transversal direction where less matter restrains the geometry to deform. Upon unclamping more freedom is brought to the system and greater distortions can occur. The component is willing to contract at the top and expand at the bottom and for this reason bending takes place together with a stress redistribution. The stress then decreases with the height of the build and can even become compressive at the top. The stress in the baseplate also evolves in the opposite way until being tensile at the bottom of the baseplate. The transition zone in-between the substrate and the first deposited layers becomes then the most critical region where the tensile stress remains maximum. These trends are observed in both WAAM and CLAD samples (Fig. 6).

\subsubsection{Comparison between processes}

The comparison in between cross sections of the deposited wall showed in Fig. 3 revealed differences in the wall geometry in the vicinity of the baseplate, top section of the wall and surface roughness. Slender wall necking in the baseplate vicinity was observed in the WAAM sample. This is link to the fact that the cooling rate is faster for the first layers leading to shorter solidification time. The deposited material has then less time to flow and the breads to flatten under gravity. This gives higher and less wide layers at the bottom of the wall. It is, however, believed that this necking can be avoided in this process by adjusting the deposition rate of the first few layers [21]. Both processes require further surface grinding and/or polishing depending on the working conditions of the potential component fabricated by these techniques. Visual investigations of the samples showed out-of-plane bending distortions of a different magnitude. The highest distortion was measured in the specimen produced by the WAAM technique $(\sim 7.2 \mathrm{~mm})$ while the CLAD sample showed $\sim 3.5 \mathrm{~mm}$ of positive bending. For technical reasons the wall were printed on Ti-baseplates of slightly different thickness (8 $\mathrm{mm}$ for the CLAD sample and $7 \mathrm{~mm}$ for the WAAM sample). Lower thickness gives weaker structure rigidity and therefore should lead to greater distortion. However, this minute difference is unlikely to be the main reason of discrepancy between the bending distortion observed (more than two times higher for the WAAM sample). The more the structure is flexible the more the system will tend to relax after unclamping. All the problem lays in finding the right balance between residual stress and distortion. The WAAM sample shows the greater distortion but also the higher tensile stress peak (observed in the longitudinal direction). This sample also showed larger fusion and heat affected zones which is likely to be linked to the greater residual stress observed. It is known that the level of residual stress and distortion achieved are linked to the inherent strain induced by thermal cycling and the volume of material that have been plastically deformed during the process [30]. It is also interesting to note that the strain in the longitudinal direction at the middle and bottom part of the wall is rather similar between the two samples. The strain in the normal direction is negative in both samples, however, the intensity is significantly stronger in the CLAD sample near the baseplate-wall transition. In the computation of stresses, this strain component $\left(\varepsilon_{z z, 103}\right)$ contributes to the compressive stress observed in the normal direction for the CLAD sample (while it is relatively null in the WAAM sample). Moreover, $\varepsilon_{\mathrm{zz}, 103}$ also affects the stress in the longitudinal direction by decreasing its value. In the middle of the samples, the stress curves show similar values and trends in both samples but approaching the top of the wall the two curves related to longitudinal direction diverge. In the case of 
the WAAM sample, a drop towards negative values is observed while the curve remains close to zero for the CLAD sample. It might be linked to the fact that the bending distortion is more important in the WAAM sample. The deformation created by the overall efforts acting internally on the structure once the sample is unclamped can lead to substantial compression at the top. Also, the last 3 to 4 layers deposited did not see the same thermal history as the other layers and some difference in the stress state can be expected. In the WAAM sample the region that has been reheated above the $\beta$-transus extends $6.5 \mathrm{~mm}$ below the sample top which also match the region where the lowest stress values were measured. In the CLAD sample, the last step of the building process is the deposition of matter on the edge of the sample (see deposition path in Fig. 1), the region located at the top of the centreline is therefore affected by this last deposition step which might contribute to the difference in stress observed. Reducing residual stresses is crucial since if they are excessive in the final component they can decrease the overall mechanical properties and further degrade the performances of the part in service. One option is to remove the baseplate since it considerably reduces the microstrain in longitudinal direction (Fig. 6c), however, depending on the design of the part and the overall desired aim this might not be a conceivable solution.

\section{Conclusions}

The main conclusions drawn from this comparative microstructure and residual stress study on the Ti-6Al-4V AM WAAM and CLAD built components are:

1. Both specimens showed fully dense structures with few pores located in the baseplate vicinity;

2. A larger $\mathrm{HAZ}$ and greater grain coarsening in the baseplate-wall region was observed in the WAAM sample;

3. In longitudinal cross sections, a tilt in the columnar growth direction was observed just in the WAAM sample;

4. Microstructures were mostly composed of narrow wavy columnar grains in the CLAD sample and large columnar grain spreading on almost all the wall height in the WAAM specimen;

5. Curved and straight layer bands were observed in the WAAM and CLAD sample, respectively. The region without layer band at the top of the specimens was larger in the WAAM sample;

6. The maximum residual stresses were observed in the wall-baseplate transition in both samples with the higher value in WAAM sample;

7. Compressive regions were detected near the wall-baseplate transition in the CLAD sample and at the top of the wall in the WAAM sample;

8. Baseplate removal noticeably reduced the microstrain in the longitudinal direction. 


\section{Acknowledgements}

The authors wish to acknowledge financial support from the AMAZE Project, which is co-funded by the European Commission in the 7th Framework Programme (contract FP7-2012-NMP-ICT-FoF313781), by the European Space Agency and by the individual partner organisations. The authors would also like to express the sincere gratitude to Prof. Moataz M. Attallah for stimulating discussions.

\section{Appendix A}

There are several methods to estimate the strain free lattice spacing $d_{0, h k l}$. Among the most used we can mention:

1. Averaging d-values over the sample height in the three principal directions [19].

2. Using the value obtained in a region near free surfaces where the strain is expected to be negligible. In the present study this would be at the top of the sample wall in transversal direction.

3. Calculating different values for $\mathrm{d}_{0, \mathrm{hk}}$ at each measured points by stress balance. This requires to impose a null value in one the principal stress directions [31] where the thickness is considered to be sufficiently small (plane approximation).

4. Measuring tiny coupons cut inside the measured sample. This technique gives the best estimation of a strain free material providing that the assembling and measurement are performed correctly to avoid any pseudo-strain issue. It is, however, destructive and requires additional beamtime (the samples cannot generally be taken out of the reactor straight after measurement for cutting due to material activation). In the case where these stress free samples are measured during a subsequent campaign, care should be taken to calibrate the measurements and account for any variation into the operating conditions (energy, beam shift, etc).

The maximum and minimum stress values comparison calculated by method 1, 2 and 3 in CLAD and WAAM samples are shown in Table 3. The averaged standard deviation observed between methods is about $75 \mathrm{MPa}$. It is worth noticing that the lowest value is always obtained using method 2. This can be explained by the fact that the strain in the transversal direction at the top of the wall may not be null but slightly positive due sample bending after unclamping. This method probably underestimate the stress and, therefore, constitutes a lower limit. It is interesting to notice that the maximum and minimum stress $\sigma_{\max }$ and $\sigma_{\min }$ in a considered sample are found exactly at the same location disregarding the method used. This shows that in the present case the choice of method used has little effect on the shape of curves and do not modify significantly the overall trend.

When samples are compared together, the first striking fact that immediately reflects is that the maximum stress is always observed for the WAAM sample independently of the method used. Another point worth mentioning is that the selected method 1 or 2 has a limited effect on the relative position of curves with respect to each other. The discrepancy is only about $2.2 \%$ of $A_{\sigma}$. This value is slightly higher for the method 3 . This is due to the fact that a different value of $d 0$. is calculated for each measured points. This method has the advantage of allowing to account for any slight variations 
of the stress-free material properties with the measurement position in the sample. It is however limited by the plane approximation made. It is indeed likely that when the sample thickness exceeds few millimetres, the stress becomes not null in the transversal direction and the assumptions made become questionable. Recent measurements performed in similar wall-samples have shown the presence of stress gradient within the wall thickness that might confirm this trend [19].

Table 3 - Comparison of the maximum and minimum stresses obtained by using three different nondestructive methods for stress-free reference estimation. $A_{\sigma}$ corresponds to the maximum amplitude measured between omax and omin which turns to be $\sigma_{\max , \text { WAAM }}-\sigma_{\min , \text { WAAM }}$ for each method.

\begin{tabular}{|c|c|c|c|}
\hline & Method 1 & Method 2 & Method 3 \\
\hline CLAD sample & & & \\
\hline $2 \theta_{0}$ & 88.0919 & 88.0581 & Variables \\
\hline$\sigma_{\min }=\sigma_{z z}$ at $Z=0$ & -249.1 & -351.1 & -210.4 \\
\hline$\sigma_{\max }=\sigma_{y y}$ at $Z=2 \mathrm{~mm}$ & 277.0 & 174.8 & 307.9 \\
\hline WAAM sample & & & \\
\hline $2 \theta 0$ & 88.0038 & 87.999 & Variables \\
\hline$\sigma_{\min }=\sigma_{y y}$ at $Z=Z_{\max }$ & -277.7 & -396.4 & -194.8 \\
\hline$\sigma_{\max }=\sigma_{y y}$ at $Z=1 \mathrm{~mm}$ & 480.9 & 361.9 & 399.8 \\
\hline Comparison between the two samples & & & \\
\hline$\left(\sigma_{\min , \text { WAAM }}-\sigma_{\min , ~ C L A D}\right)$ & -28.6 & -45.3 & 15.6 \\
\hline$\left(\sigma_{\min , \text { WAAM }}-\sigma_{\min , \text { CLAD }}\right) \mathrm{A}_{\sigma}$ & $-3.8 \%$ & $-6.0 \%$ & $2.6 \%$ \\
\hline$\left(\sigma_{\text {max }, \text { WAAM }}-\sigma_{\max , \text { CLAD }}\right)$ & 203.9 & 187.1 & 91.9 \\
\hline$\left(\sigma_{\max }\right.$, WAAM $\left.-\sigma_{\max }, \mathrm{CLAD}\right) \mathrm{A}_{\sigma}$ & $26.9 \%$ & $24.7 \%$ & $15.5 \%$ \\
\hline
\end{tabular}

\section{References}

[1] S.L. Semiatin, V. Seetharaman, I. Weiss, The thermomechanical processing of alpha/ beta titanium alloys, J. Metals 49 (6) (1997) 33-39.

[2] H.B. Qi, D.L. Ren, M.J. Zheng, Research on forming technology of electron beam selective melting for Ti-6Al-4V powder, Appl. Mech. Mater. 44-47 (2011) 2778-2782.

[3] H.M. Flower, High Performance Materials in Aerospace, Chapman and Hall, London, UK, 1995.

[4] B. Baufeld, O. Van der Biest, R. Gault, Additive manufacturing of Ti-6Al-4V components by shape metal deposition: microstructure and mechanical properties, Mater. Des. 31 (2010) S106-S111.

[5] P.A. Colegrove, H.E. Coules, J. Fairman, F. Martina, T. Kashoob, H. Mamash, L.D. Cozzolino, Microstructure and residual stress improvement in wire and arc additively manufactured parts through high-pressure rolling, J. Mater. Process. Technol. 213 (2013) 1782-1791. 
[6] P. Kazanas, P. Deherkar, P. Almeida, H. Lockett, S. Williams, Fabrication of geometrical features using wire and arc additive manufacture, Proc. Inst. Mech. Eng. B J. Eng. Manuf. 226 (6) (2012) 1042-1051.

[7] W. He, J. Wenpeng, L. Haiyan, T. Huiping, K. Xinting, Y. Huang, Research on preheating of titanium alloy powder in electron beam melting technology, Rare Metal Mater. Eng. 40 (12) (2011) 2072-2075.

[8] F. Weng, C. Chen, Y. Huijun, Research status of laser cladding on titanium and its alloys: a review, Mater. Des. 58 (0) (2014) 412-425.

[9] X. Gong, T. Anderson, K. Chou, Review on powder-based electron beam additive manufacturing technology, Manuf. Rev. 1 (2) (2014).

[10] Y. Zhaia, H. Galarraga, D.A. Lados, Microstructure evolution, tensile properties, and fatigue damage mechanisms in Ti-6Al-4V alloys fabricated by two additive manufacturing techniques, Procedia Eng. 114 (2015) 658-666.

[11] H. Gong, K. Rafi, H. Gu, G.D.J. Ram, T. Starr, B. Stucker, Influence of defects on mechanical properties of Ti-6Al-4V components produced by selective laser melting and electron beam melting, Mater. Des. 86 (2015) 545-554.

[12] T. Schenk, I.M. Richardson, M. Kraska, S. Ohnimus, A study on the influence of clamping on welding distortion, Comput. Mater. Sci. 45 (4) (2009) 999-1005.

[13] R.J. Moat, A.J. Pinkerton, L. Li, P.J. Withers, M. Preuss, Residual stresses in laser direct metal deposited Waspaloy, Mater. Sci. Eng. A 528 (2011) 2288-2298.

[14] P. M. Sequeira, P. Almeida, S. Williams, Proc. Innovative process model of Ti-6Al-4V additive layer manufacturing using cold metal transfer (CMT), 21st International Solid Freeform Fabrication Symposium, August 9-11, Austin, TX, 2010.

[15] P.A. Kobryn, S.L. Semiatin, The laser additive manufacture of Ti-6AI-4V, JOM 53 (9) (2001) 40 42.

[16] A. Syed, H. Kabir, X. Cao,J . Gholipour, P. Wanjara, J. Cuddy, A. Birur, M. Medraj, Effect of post weld heat treatment on microstructure, hardness, and tensile properties of laser-welded Ti-6Al4V, Metall. Mater. Trans. A 43 (11) (2012) 4171-4184.

[17] N.S. Rossini, M. Dassisti, K.Y. Benyounis, A.G. Olabi, Methods of measuring residual stresses in components, Mater. Des. 35 (2012) 572-588.

[18] M.T. Hutchings, A.D. Kravitz, Measurement of Residual and Applied Stress Using Neutron Diffraction, Kluwer Acad. Publ, Dordrecht, Netherlands, 1992.

[19] P.A. Colegrove, F. Martina, M.J. Roy, B.A. Szost, S. Terzi, S.W. Williams Stewart, P.J. Withers, D.J. Jarvis, High pressure interpass rolling of wire + arc additively manufactured titanium components, Adv. Mater. Res. 996 (2014) 694-700.

[20] A.M. Stapleton, S.L. Raghunathan, I. Bantounas, H.J. Stone, T.C. Lindley, D. Dye, Evolution of lattice strain in Ti-6Al-4V during tensile loading at room temperature, Acta Mater. 56 (20) (2008) 6186-6196.

[21] F. Martina, J. Mehnen, S.W. Williams, P. Colegrove, F. Wang, Investigation of the benefits of plasma deposition for the additive layer manufacture of Ti-6Al-4V, J. Mater. Process. Technol. 212 (6) (2012) 1377-1386 (00025).

[22] T.I. Deb Roy, S.A. David, Physical processes in fusion welding, Rev. Mod. Phys. 67(1) (1995) 85. 
[23] W. Kurz, C. Bezencon, M. Gaumann, Columnar to equiaxed transition in solidification processing, Sci. Technol. Adv. Mater. 2 (1) (2001) 185-191.

[24] R.J. Moat, A.J. Pinkerton, L. Li, P.J. Withers, M. Preuss, Crystallographic texture and microstructure of pulsed diode laser-deposited waspaloy, Acta Mater. 57 (4) (2009) 1220-1229.

[25] S.M. Kelly, S.L. Kampe, Microstructural evolution in laser-deposited multilayer Ti-6Al-4V builds: part I. microstructural characterisation, Metall. Mater. Trans. A 35 (6) (2004) 1861-1867.

[26] M.Shunmugavel, A. Polishetty, G. Littlefair, Microstructure and mechanical properties of wrought and additive manufactured Ti-6Al-4V cylindrical bars, Procedia Technol. 20 (2015) 231-236.

[27] Y. Yu, F. Li Chenglin, H.S. Yanyan, Y. Wenjun, Effect of trace boron addition on microstructure and properties of as-cast Ti-6Al-4V alloy, Rare Metal Mater. Eng. 43 (2014) 2908-2911.

[28] F. Wang,S. Williams, P. Colegrove, A.A. Antonysamy, Microstructure and mechanical properties of wire and arc additive manufactured Ti-6Al-4V, Metall. Mater. Trans. A 44 (2) (February 2013) 968-977.

[29] J.A. Dantzig, M. Rappaz, Solidification, EPFL press, 2009.

[30] Y. Ueda,H. Murakawa, N. Ma, Welding Deformation and Residual Stress Prevention, Elsevier Science, 2012.

[31] M.M. Attallah, M. Preuss, C. Boonchareon, A. Steuwer, J.E. Daniels, D.J. Hughes, C. Dungey, G.J. Baxter, Microstructural and residual stress development due to inertia friction welding in Ti6246, Metall. Mater. Trans. A 41A (6) (2010) 3149-3161. 
2015-09-28

\section{A comparative study of additive} manufacturing techniques: Residual stress and microstructural analysis of CLAD and WAAM printed Ti-6Al-4V components

Szost, Blanka A.

Elsevier

Szost B, Terzi S, Martina F, et al., A comparative study of additive manufacturing techniques:

Residual stress and microstructural analysis of CLAD and WAAM printed Ti-6Al-4V components. Materials and Design, Volume 89, January 2016, pp. 559-567

https://doi.org/10.1016/j.matdes.2015.09.115

Downloaded from Cranfield Library Services E-Repository 